\title{
Impulse Based Substructuring for Coupling Offshore Structures and Wind Turbines in Aero-Elastic Simulations
}

\author{
P.L.C. van der Valk* and D.J. Rixen* \\ Delft University of Technology, Delft, 2628CD, The Netherlands
}

\begin{abstract}
In order to achieve the goal of $20 \%$ renewable energy in 2020 , as set by the European Union, large offshore wind farms are either under construction or in development throughout Europe. As many of the "easy" locations are already under development, offshore wind farms are moving further offshore into deeper waters, which results in a set of new technical challenges. One of these challenges comes from the fact that the traditional solution of placing monopile-foundations is possibly no longer sufficient to anchor the latest generation of wind turbines to the seabed. This requires that different and more complex types of offshore structures, such as jackets, are used as foundations. Appropriate models for these more complex foundations, are often not available in aero-elastic simulation software. The current design practices, such as equivalent interface stiffness and mass matrices for the offshore structure, could lead to errors in the coupled simulations due to dynamics that are not modeled. In this paper an alternative method, Impulse Based Substructuring, is proposed to efficiently and accurately include the dynamic behavior of the support structure in the load simulations. The method is demonstrated using the NREL 5MW reference turbine and UpWind reference jacket. It is shown that the method is able to accurately compute the coupled dynamics and requires only a number of small augmentations to the standard Newmark time integration scheme for nonlinear finite element models.
\end{abstract}

\section{Introduction}

Present day media are full of items on peak oil, rising oil prices, climate changes, $\mathrm{CO}_{2}$ reductions and our continuously growing energy needs. More sustainable ways of generating power on an industrial scale need to be implemented, simply because the currently used resources will someday be exhausted, or become unaffordable. The Europe Union has acknowledged this fact, therefore setting a target of $20 \%$ renewable energy in 2020. One of the more promising ways of generating renewable electricity on a large scale for achieving this goal is provided by wind energy. As many of the favorable onshore locations are usually also densely populated and therefore not suited for large wind farms, much of these farms will have to be built offshore.

Since a lot of the shallow, near-shore sites have already been built or are under development, the current trend is to go to deeper waters which are usually also further offshore. Combining this trend with the ever increasing sizes of offshore wind turbines, gives that the traditional offshore solution, the monopile, is often no longer (economically and technically) feasible. Therefore, more complex offshore structures, such as tripods and jackets, are needed for the installation of these offshore wind turbines. However, combining these more complex structures with the aero-elastic wind turbine models leads to some challenges, as the models of these offshore structures generally consist of significantly more degrees of freedom (DoF) and thus lead to a huge increase in computational effort. In addition to this, there is often a clear design split between the foundation designer and the turbine manufacturer, who are both reluctant to share their detailed models as that would infringe on their intellectual properties. The most common approach to overcome these issues is to apply Guyan reduction in order to create a superelement ${ }^{1,2}$ of the offshore foundation model. This reduction method statically reduces the (linearized) offshore structure to its interface with the turbine, hence decreasing the number of DoF to only six. But what is often disregarded is that this leads to a very crude

*Faculty of Mechanical, Maritime, and Materials Engineering, Section of Engineering Dynamics, Mekelweg 2 
simplification of the reality, as all of the internal dynamic behavior of the offshore structure and almost all potential coupling between the dynamics of the offshore structure and turbine is neglected. By not including this behavior in the models, the loading and hence the cumulative damage on both the turbine and offshore structure can be significantly underestimated or overestimated. ${ }^{3}$

In this paper an alternative method is presented for coupling the wind turbine to the offshore structure in aero-elastic simulations. The method, named Impulse Based Substructuring, ${ }^{4}$ uses the impulse responses of the offshore structure in order to create a substructure that includes all the (internal) dynamic behavior of the offshore structure. As the impulse response functions are basically input-output relations, the number of degrees of freedom of the model can be reduced simply to the number of inputs and outputs required. Firstly, section II will present some of the basics about Impulse Response Functions and how to obtain and use them. In section III the theory behind the method will be briefly explained and it will be shown how to assembly a nonlinear finite element model to an impulse response function in order to compute the coupled behavior. If one wants to perform simulations that start from a nonzero initial condition, one needs to solve an initial nonlinear static problem, which is presented in section IV. In section V the method will be applied for coupling the NREL 5MW reference turbine to the UpWind reference jacket, thus demonstrating its accuracy. Finally, in section VI the conclusions are presented.

\section{Time Integration using Impulse Response Functions}

As the name already implies, Impulse Response Functions (IRFs) show the response of the system at hand excited by unit impulse force. Hence, they give the input-output relationship of a system or structure. As any force in time can be seen as a succession of impulses over time, ${ }^{5}$ IRFs can be used to determine the time response of a system. In this section it will be shown how to obtain and use these IRFs for time integration.

\section{A. Time integration using the impulse response functions}

Firstly, starting from the equation of motion of a linear(-ized) dynamic system:

$$
\boldsymbol{M} \ddot{\boldsymbol{u}}(t)+\boldsymbol{C} \dot{\boldsymbol{u}}(t)+\boldsymbol{K} \boldsymbol{u}(t)=\boldsymbol{f}(t) .
$$

Here, $\boldsymbol{M}$ denotes the mass matrix, $\boldsymbol{C}$ the damping matrix, $\boldsymbol{K}$ the stiffness matrix, $\boldsymbol{f}(t)$ the external force, which is a function of time and $\boldsymbol{u}(t), \dot{\boldsymbol{u}}(t), \ddot{\boldsymbol{u}}(t)$, respectively the displacements, velocities and accelerations, which are also a function of time. In the frequency domain $\ddot{\boldsymbol{u}}(\omega)=i \omega \dot{\boldsymbol{u}}(\omega)=-\omega^{2} \boldsymbol{u}(\omega)$, and the equations can be written in the form

$$
\left(-\omega^{2} \boldsymbol{M}+i \omega \boldsymbol{C}+\boldsymbol{K}\right) \boldsymbol{u}(\omega)=\boldsymbol{Z}(\omega) \boldsymbol{u}(\omega)=\boldsymbol{f}(\omega)
$$

The equations of motion are transformed from the time to the frequency domain in Eq. (2), where $\boldsymbol{Z}(\omega)$ is the dynamic stiffness matrix, which is the inverse of the dynamic flexibility matrix $\boldsymbol{Y}(\omega)$, as shown in Eq. (3).

$$
\boldsymbol{Z}(\omega)^{-1} \boldsymbol{f}(\omega)=\boldsymbol{Y}(\omega) \boldsymbol{f}(\omega)=\boldsymbol{u}(\omega)
$$

So, if one would be interested in the time response of a system caused by a certain input force, Eq. (3) can be transformed back to the time domain via an inverse Fourier transform, which would result in:

$$
\boldsymbol{u}(t)=\int_{\tau=0}^{t} \boldsymbol{Y}(t-\tau) \boldsymbol{f}(\tau) d \tau,
$$

where, $\boldsymbol{Y}(t)$ is the impulse response function of the dynamic system at hand. This integral equation is known as a convolution product or Duhamel integral, and can be discretized in order to solve it in a numerical way.

\section{B. Discretization of a convolution product}

The obtained convolution integral, given in Eq. (4), is a continuous equation and needs to be discretized in order to be used for numerical computations. By regarding the applied force $\boldsymbol{f}(t)$ as a sum of discrete impulses, one could interpret this integral as being a sum of the responses to impulse excitations between 
$t=0$ and $t=\tau$. In general however, the analytical impulse response function is not available, therefore one can compute a discrete numerical approximation $\boldsymbol{Y}\left(t_{n}\right)$, for $t_{n}=0 \ldots t_{n_{\max }}$. Due to this, the integral in Eq. (4) is replaced by a finite sum, which converges to the exact solution as $d t$ goes to zero.

$$
\boldsymbol{u}_{n}=d t \sum_{i=0}^{n-1} \boldsymbol{Y}_{n-i} \boldsymbol{f}_{i}
$$

Note that the sum in Eq. (5) is from 0 to $n-1$, since $\boldsymbol{Y}_{0}=\boldsymbol{O}$. This is due to the fact that the displacement response at $t=0$ to an impulse at $t=0$ is zero. In this sum, it is assumed that both the impulse response function and the forcing function are piecewise constant. A more accurate approximation would be to assume a piecewise linear variation of the forcing function a the different time stations, which would result in the following sum.

$$
\boldsymbol{u}_{n}=\frac{d t}{2} \sum_{i=0}^{n-1} \boldsymbol{Y}_{n-i}\left(\boldsymbol{f}_{i}+\boldsymbol{f}_{i+1}\right)
$$

Although this discretization is more accurate than the one given in Eq. (5), it still only allows to compute the response of a system initially at rest.

\section{Computing the response from nonzero initial conditions}

The response of a linear system to an external force, starting from a set of non-zero initial conditions, can be regarded as a superposition of a dynamic response $(\boldsymbol{v}(t))$ and the initially applied displacements $\left(\boldsymbol{u}_{0}\right)$.

$$
\boldsymbol{u}(t)=\boldsymbol{v}(t)+\boldsymbol{u}_{0}
$$

Substituting Eq. (7) into the linear equations of motion of a general dynamic system, gives

$$
\boldsymbol{M} \ddot{\boldsymbol{v}}(t)+\boldsymbol{C} \dot{\boldsymbol{v}}(t)+\boldsymbol{K} \boldsymbol{v}(t)=\boldsymbol{f}(t)-\boldsymbol{K} \boldsymbol{u}_{0},
$$

where we change the variable from $\boldsymbol{u}(t)$ to $\boldsymbol{v}(t)$. As discussed in subsection B, this can be rewritten into a discretized convolution product

$$
\boldsymbol{u}_{n}=\boldsymbol{u}_{0}+\frac{d t}{2} \sum_{i=0}^{n-1} \boldsymbol{Y}_{n-i}\left(\tilde{\boldsymbol{f}}_{i}+\tilde{\boldsymbol{f}}_{i+1}\right)
$$

where,

$$
\tilde{\boldsymbol{f}}_{i}=\boldsymbol{f}_{i}-\boldsymbol{K} \boldsymbol{u}_{0}
$$

A brief introduction to discrete time integration using the impulse response matrices and the convolution product has been given. In the next sections these methods will be used for deriving the Impulse Based Substructuring method.

\section{Computing the Unit Impulse Response Functions}

For computing the unit impulse force response functions, the Newmark time integration scheme is used. There are several initial conditions one can use to compute the impulse response functions. ${ }^{4}$ In this paper it is assumed that a unit impulse is given in the first time step $\left(t_{0}=0\right)$, this is chosen as it is consistent with the constant average Newmark method. From this assumption, the following initial conditions can be derived

$$
\begin{aligned}
& \boldsymbol{u}_{0}=0 \\
& \dot{\boldsymbol{u}}_{0}=0 \\
& \ddot{\boldsymbol{u}}_{0}=\boldsymbol{M}^{-1} \frac{\mathbf{1}_{j}}{d t},
\end{aligned}
$$

where $\mathbf{1}_{j}$ is a vector with a unit coefficient for $\operatorname{dof} j$. Note that, throughout this paper the constant average acceleration variant of the Newmark $(\gamma=0.5, \beta=0.25)$ is used. This thus implies that the values of the forcing function vary linearly between the different time stations, as can be seen in figure 1 . Due to this, the system with the initial conditions given in Eq. (10) is in fact excited not by a unit pulse, but by a half 


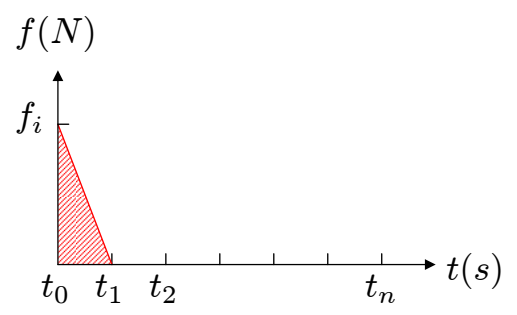

Figure 1. Force impulse in the constant average acceleration Newmark scheme

unit pulse and the final result has to be corrected for this. Using these initial conditions and performing a time simulation, one is able to obtain the accelerations, velocities and displacements of all DoF as a result of the impulse at DoF $j$. Thereby determining the $j^{\text {th }}$ column of the Unit Impulse Response Matrix, which is shown for displacements here. Hence, computing the response of the system to an initial impulse on each degree of freedom where a force is applied (inputs), the entire set of IRFs, as needed in Eq. (6), are found. They can be written in a matrix form as:

$$
\boldsymbol{Y}\left(t_{n}\right)=\left[\begin{array}{ccccc}
Y_{11}\left(t_{n}\right) & \ldots & Y_{1 j}\left(t_{n}\right) & \ldots & Y_{1 N}\left(t_{n}\right) \\
\vdots & \ddots & \vdots & \ddots & \vdots \\
Y_{j 1}\left(t_{n}\right) & \ldots & Y_{j j}\left(t_{n}\right) & \ldots & Y_{j N}\left(t_{n}\right) \\
\vdots & \ddots & \vdots & \ddots & \vdots \\
Y_{N 1}\left(t_{n}\right) & \ldots & Y_{N j}\left(t_{n}\right) & \ldots & Y_{N N}\left(t_{n}\right)
\end{array}\right]
$$

\section{E. Using an impulse response due to a distributed load}

In engineering practice however, the offshore foundations is excited by waves and currents on a large part of the structure. If this is now seen from a modeling perspective, this area will consist of a large number of nodes in a finite element model, and the number of entries in the matrix containing the required IRFs would grow quadratically with respect to the size of the force loaded area. In order to overcome this issue, we describe these forces as a summation of space distributions of the force $\left(\boldsymbol{\Phi}_{i}(\boldsymbol{x})\right)$, multiplied by their corresponding force amplitudes $\left(\eta_{i}(t)\right)$ :

$$
\boldsymbol{f}(\boldsymbol{x}, t)=\sum_{i=1}^{n_{\phi}} \boldsymbol{\Phi}_{i}(\boldsymbol{x}) \eta_{i}(t)=\boldsymbol{\Phi} \boldsymbol{\eta}
$$

As can be seen, separation of variables is applied to the force $\boldsymbol{f}(x, t)$, which is dependent on both time $(t)$ and location $(\boldsymbol{x})$. By substituting Eq. (12) into Eq. (4), the impulse response describes the response of a structure due to a distributed load.

$$
\boldsymbol{u}(t)=\int_{0}^{t} \boldsymbol{Y}(t-\tau) \boldsymbol{\Phi}(\boldsymbol{x}) \boldsymbol{\eta}(\tau) d \tau
$$

Suppose that the system contains a large number $(p)$ of force loaded dof (input) with only $N$ response dof of interest (output), such that $N<<p$. In Eq. (13) $\boldsymbol{Y}(t)$ is a $N \times p \times n_{\max }$ sized matrix (where $n_{\max }$ is the number of time steps), hence computing the needed IRFs and evaluating the convolution integral will be computationally expensive. Using the shape distributions of the load to reduce the size of the impulse response function, leads to:

$$
\boldsymbol{u}(t)=\int_{0}^{t} \boldsymbol{Y}_{\boldsymbol{\Phi}}(t-\tau)(\boldsymbol{x}) \boldsymbol{\eta}(\tau) d \tau
$$

where,

$$
\boldsymbol{Y}_{\boldsymbol{\Phi}}(t)=\boldsymbol{Y}(t) \boldsymbol{\Phi}
$$

This step reduces the size of the impulse response function from $N \times p \times n_{\max }$ to $N \times p_{\phi} \times n_{\max }$, where $p_{\phi}$ equals the number of force shape distribution functions and $p_{\phi}<<p$. This approach will be used in order to incorporate the wave loading on the jacket structure in the coupled load simulations. 


\section{The Impulsed Based Substructuring Method}

As the goal of this work is to combine the offshore structure, which is given as a set of IRFs, and the wind turbine, which is a nonlinear FE model, a method had to be found to assemble these two in order to obtain the coupled dynamic behavior. Firstly, the necessary conditions for assembly are discussed and secondly it is shown how to rework the equations in order to obtain the coupled set of equations and finally, a flowchart is given which summarizes the method.

\section{A. Conditions for assembly: Compatibility and Equilibrium}

Starting from the set of uncoupled equations, where the variables with superscript $(s)$ denote the offshore structure, which is described in its IRFs and the variables with superscript $(r)$ denote the wind turbine, which is given as a (non-) linear FE model and $\boldsymbol{p}^{(r)}\left(\dot{\boldsymbol{u}}_{n}^{(r)}, \boldsymbol{u}_{n}^{(r)}\right)$ is the nonlinear force vector which contains all the aerodynamic forces and possibly other nonlinear stiffness and damping contributions. ${ }^{a}$ In case of an operational turbine, the rotor will rotate and hence, the mass matrix $\boldsymbol{M}^{(r)}\left(\boldsymbol{u}_{n}^{(r)}\right)$ also becomes nonlinear.

$$
\left\{\begin{array}{l}
\boldsymbol{u}_{n}^{(s)}=\boldsymbol{u}_{0}^{(s)}+\frac{d t}{2} \sum_{i=0}^{n-1} \boldsymbol{Y}_{n-i}^{(s)}\left(\tilde{\boldsymbol{f}}_{i}^{(s)}+\tilde{\boldsymbol{f}}_{i+1}^{(s)}-\boldsymbol{g}_{i}^{(s)}-\boldsymbol{g}_{i+1}^{(s)}\right) \\
\boldsymbol{M}^{(r)}\left(\boldsymbol{u}_{n}^{(r)}\right) \ddot{\boldsymbol{u}}_{n}^{(r)}+\boldsymbol{p}^{(r)}\left(\dot{\boldsymbol{u}}_{n}^{(r)}, \boldsymbol{u}_{n}^{(r)}\right)=\boldsymbol{f}_{n}^{(r)}-\boldsymbol{g}_{n}^{(r)}
\end{array}\right.
$$

Here, $\tilde{\boldsymbol{f}}_{i}^{(s)}=\boldsymbol{f}_{i}^{(s)}-\boldsymbol{K}^{(s)} \boldsymbol{u}_{0}^{(s)}$ and $\boldsymbol{g}_{n}$ denotes the unknown interface forces between the offshore structure and the wind turbine, which will ensure the coupling between the two substructures. In order to obtain a fully coupled set of equations, two conditions have to be satisfied. ${ }^{6}$ The first one being compatibility, which means that the displacements of the interface nodes of both structures have to be equal, which thus means that no gap is allowed between the two substructures.

$$
\boldsymbol{u}_{[b]}^{(s)}=\boldsymbol{u}_{[b]}^{(r)},
$$

where the subscript $[b]$ denotes the interface (or boundary) nodes. The compatibility condition can also be written using signed Boolean operators that work on the boundary DoF within the full set of DoF, such that

$$
\left[\begin{array}{ll}
\boldsymbol{B}^{(s)} & \boldsymbol{B}^{(r)}
\end{array}\right]\left[\begin{array}{l}
\boldsymbol{u}^{(s)} \\
\boldsymbol{u}^{(r)}
\end{array}\right]=\boldsymbol{B} \boldsymbol{u}=\boldsymbol{O}
$$

The second condition is Equilibrium, which states that the sum of the forces at the connecting nodes have to be equal to zero, such that no resulting force between the substructures exists.

$$
\boldsymbol{g}_{[b]}^{(s)}+\boldsymbol{g}_{[b]}^{(r)}=\boldsymbol{0}
$$

This means that the interface forces have to be opposite in sign and equal in magnitude. Using the signed Boolean operator $\boldsymbol{B}$ from Eq. (18), this relation can be written as:

$$
\left[\begin{array}{l}
\boldsymbol{g}^{(s)} \\
\boldsymbol{g}^{(r)}
\end{array}\right]=-\boldsymbol{B}^{T} \boldsymbol{\lambda},
$$

where $\boldsymbol{\lambda}$ are known as Lagrange Multipliers that denote the magnitudes of the interface forces. Hence, by using Eq. (20), the number of unknowns is reduced. Substitution of both the compatibility condition, given in Eq. (18), and the equilibrium condition, as given in Eq. (20), into Eq. (16), results in the set of coupled equations.

$$
\left\{\begin{array}{l}
\boldsymbol{u}_{n}^{(s)}=\boldsymbol{u}_{0}^{(s)}+\frac{d t}{2} \sum_{i=0}^{n-1} \boldsymbol{Y}_{n-i}^{(s)}\left(\tilde{\boldsymbol{f}}_{i}^{(s)}+\tilde{\boldsymbol{f}}_{i+1}^{(s)}-\boldsymbol{B}^{(s)^{T}}\left(\boldsymbol{\lambda}_{i}+\boldsymbol{\lambda}_{i+1}\right)\right) \\
\boldsymbol{M}^{(r)}\left(\boldsymbol{u}_{n}^{(r)}\right) \ddot{\boldsymbol{u}}_{n}^{(r)}+\boldsymbol{p}^{(r)}\left(\dot{\boldsymbol{u}}_{n}^{(r)}, \boldsymbol{u}_{n}^{(r)}\right)=\boldsymbol{f}_{n}^{(r)}-\boldsymbol{B}^{(r)^{T}} \boldsymbol{\lambda}_{n} \\
\boldsymbol{B} \boldsymbol{u}_{n}=\boldsymbol{O}
\end{array}\right.
$$

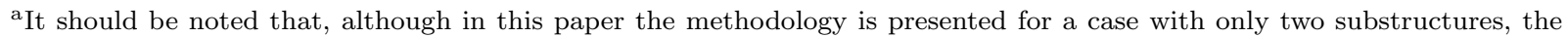
approach is general and can easily be extended to more substructures. 
In the next section, it will be discussed how to rewrite these equations in order to solve them in a time stepping scheme.

\section{B. Solving the coupled set of equations}

As the goal is to develop a coupled time integration scheme, an adequate time discretization for the wind turbine substructure has to be applied. As the Newmark time discretization ${ }^{7}$ is the most common for computing the time response of a (nonlinear) finite element model, it will also be applied for the current method. In the Newmark scheme, a Taylor expansion is used to deduce the velocities and displacements of the next time station, according to:

$$
\begin{aligned}
& \dot{\boldsymbol{u}}_{n}=\dot{\boldsymbol{u}}_{n-1}+(1-\gamma) d t \ddot{\boldsymbol{u}}_{n-1}+\gamma d t \ddot{\boldsymbol{u}}_{n}=\dot{\hat{\boldsymbol{u}}}_{n}+\gamma d t \ddot{\boldsymbol{u}}_{n} \\
& \boldsymbol{u}_{n}=\boldsymbol{u}_{n-1}+d t \dot{\boldsymbol{u}}_{n-1}+d t^{2}\left(\frac{1}{2}-\beta\right) \ddot{\boldsymbol{u}}_{n-1}+d t^{2} \beta \ddot{\boldsymbol{u}}_{n}=\hat{\boldsymbol{u}}_{n}+d t^{2} \beta \ddot{\boldsymbol{u}}_{n},
\end{aligned}
$$

where $\dot{\hat{\boldsymbol{u}}}_{n}$ and $\hat{\boldsymbol{u}}_{n}$ are referred to as the predictors and are depending on the displacements, velocities and accelerations of the previous time step. As the system of equations of motion, given in Eq. (21), for the turbine is assumed to be nonlinear, they can be written as

$$
\boldsymbol{r}_{n}^{(r)}\left(\ddot{\boldsymbol{u}}_{n}^{(r)}, \dot{\boldsymbol{u}}_{n}^{(r)}, \boldsymbol{u}_{n}^{(r)}, \boldsymbol{\lambda}_{n}\right)=\boldsymbol{M}^{(r)}\left(\boldsymbol{u}_{n}^{(r)}\right) \ddot{\boldsymbol{u}}_{n}^{(r)}+\boldsymbol{p}^{(r)}\left(\dot{\boldsymbol{u}}_{n}^{(r)}, \boldsymbol{u}_{n}^{(r)}\right)+\boldsymbol{B}^{(r)^{T}} \boldsymbol{\lambda}_{n}-\boldsymbol{f}_{n}^{(r)}=\boldsymbol{O} .
$$

Here, $\boldsymbol{r}_{n}^{(r)}$ is the residual force vector and can be regarded as an error on the equilibrium, which should be made equal to zero. By inverting the original Newmark relations (22) we obtain

$$
\begin{aligned}
\ddot{\boldsymbol{u}}_{n}^{(r)} & =\frac{1}{d t^{2} \beta}\left(\boldsymbol{u}_{n}^{(r)}-\hat{\boldsymbol{u}}_{n}^{(r)}\right) \\
\dot{\boldsymbol{u}}_{n}^{(r)} & =\dot{\hat{\boldsymbol{u}}}_{n}^{(r)}+\frac{\gamma}{d t \beta}\left(\boldsymbol{u}_{n}^{(r)}-\hat{\boldsymbol{u}}_{n}^{(r)}\right),
\end{aligned}
$$

where both the accelerations and velocities are written as a function of the displacements only. By substituting Eq. (24) into Eq. (23), the residual becomes a function of the displacements and interface forces.

$$
\boldsymbol{r}_{n}^{(r)}\left(\boldsymbol{u}_{n}^{(r)}, \boldsymbol{\lambda}_{n}\right)=\boldsymbol{O}
$$

In order to minimize this nonlinear set of equations, we would like to solve it using Newton-Raphson iterations, while only iterating on the displacements and not the interface forces. Hence, it is required to find an explicit relation between the set of displacements and the interface forces. Starting from the first line of Eq. (21), the response can be split in a known part (analog to the "predictor" used for Newmark) and an unknown part,

$$
\boldsymbol{u}_{n}^{(s)}=\tilde{\boldsymbol{u}}_{n}^{(s)}-\frac{d t}{2} \boldsymbol{Y}_{1}^{(s)} \boldsymbol{B}^{(s)^{T}} \boldsymbol{\lambda}_{n}
$$

where,

$$
\tilde{\boldsymbol{u}}_{n}^{(s)}=\boldsymbol{u}_{0}^{(s)}+\frac{d t}{2} \sum_{i=0}^{n-2} \boldsymbol{Y}_{n-i}^{(s)}\left(\tilde{\boldsymbol{f}}_{i}^{(s)}+\tilde{\boldsymbol{f}}_{i+1}^{(s)}-\boldsymbol{B}^{(s)^{T}}\left(\boldsymbol{\lambda}_{i}+\boldsymbol{\lambda}_{i+1}\right)\right)+\frac{d t}{2} \boldsymbol{Y}_{1}^{(s)}\left(\tilde{\boldsymbol{f}}_{n-1}^{(s)}+\tilde{\boldsymbol{f}}_{n}^{(s)}-\boldsymbol{B}^{(s)^{T}} \boldsymbol{\lambda}_{n-1}\right)
$$

Substituting this into the compatibility equation, given in Eq. (18)

$$
\left[\begin{array}{ll}
\boldsymbol{B}^{(s)} & \boldsymbol{B}^{(r)}
\end{array}\right]\left[\begin{array}{l}
\tilde{\boldsymbol{u}}_{n}^{(s)}-\frac{d t}{2} \boldsymbol{Y}_{1}^{(s)} \boldsymbol{B}^{(s)^{T}} \boldsymbol{\lambda}_{n} \\
\boldsymbol{u}_{n}^{(r)}
\end{array}\right]=\boldsymbol{O},
$$

and rewriting this, gives us an explicit equation for $\boldsymbol{\lambda}_{n}$.

$$
\boldsymbol{\lambda}_{n}=\left(\boldsymbol{B}^{(s)} \boldsymbol{Y}_{1}^{(s)} \boldsymbol{B}^{(s)^{T}}\right)^{-1}\left[\begin{array}{ll}
\boldsymbol{B}^{(s)} & \boldsymbol{B}^{(r)}
\end{array}\right]\left[\begin{array}{c}
\tilde{\boldsymbol{u}}_{n}^{(s)} \\
\boldsymbol{u}_{n}^{(r)}
\end{array}\right],
$$




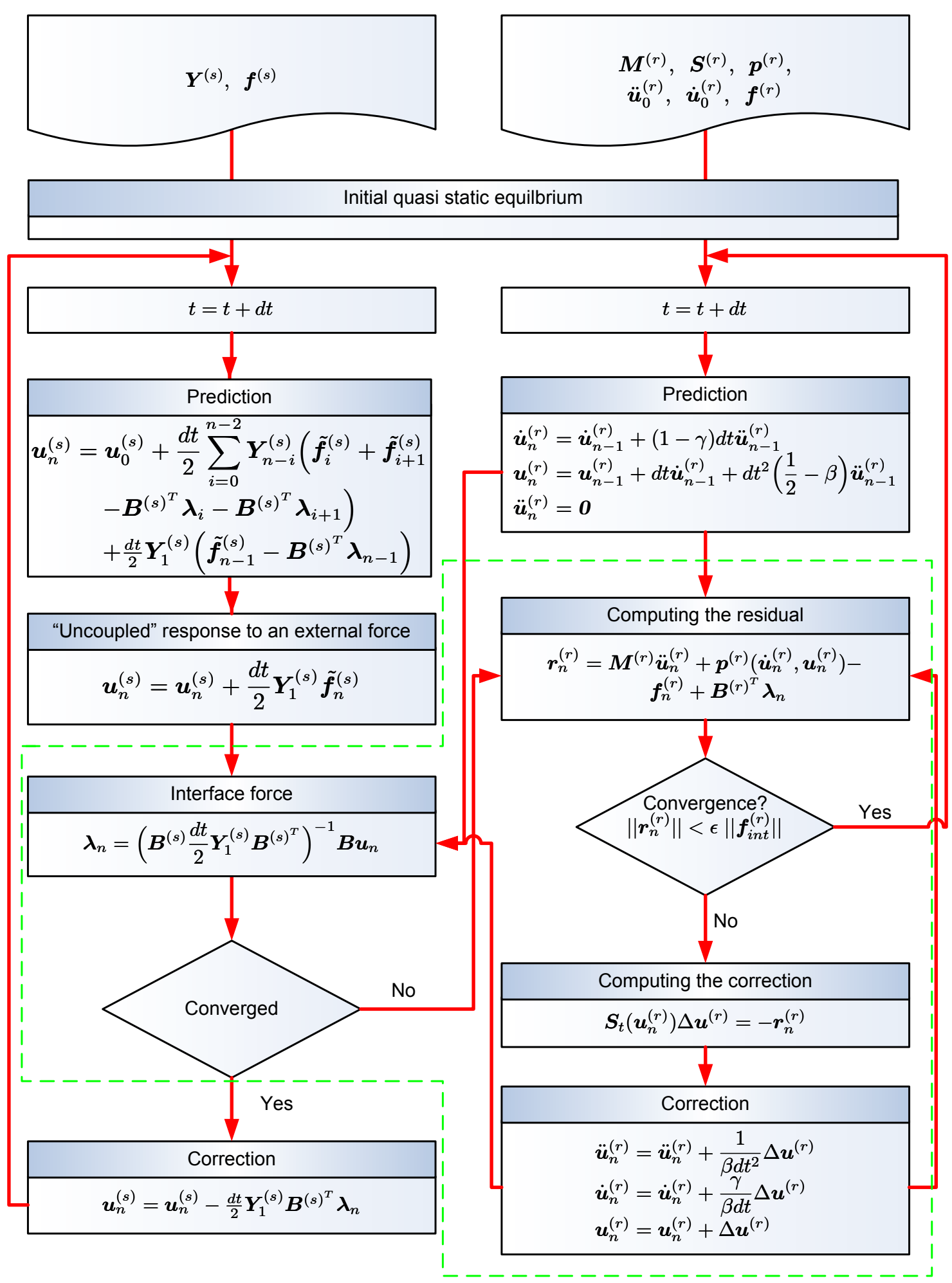

Figure 2. Integration scheme for the non-linear IBS case

Substituting Eq. (29) into Eq. (23) gives a direct (non-linear) relation between the residual and the set of displacements.

$$
\boldsymbol{r}_{n}^{(r)}\left(\boldsymbol{u}_{n}^{(r)}\right)=\boldsymbol{O}
$$


This is the non-linear problem, that will be minimized using Newton-Raphson iterations. Hence we successively solve the following set of linear equations

$$
\boldsymbol{S}\left(\boldsymbol{u}_{n}^{(r)}\right) \Delta \boldsymbol{u}^{(r)}=-\boldsymbol{r}_{n}^{(r)},
$$

where, $\boldsymbol{S}\left(\boldsymbol{u}_{n}^{(r)}\right)$ is the iteration matrix. Using the computed $\Delta \boldsymbol{u}^{(r)}$ the accelerations, velocities and displacements are updated.

$$
\begin{aligned}
& \ddot{\boldsymbol{u}}_{n}^{(r)}=\ddot{\boldsymbol{u}}_{n}^{(r)}+\frac{1}{\beta d t^{2}} \Delta \boldsymbol{u}^{(r)} \\
& \dot{\boldsymbol{u}}_{n}^{(r)}=\dot{\boldsymbol{u}}_{n}^{(r)}+\frac{\gamma}{\beta d t} \Delta \boldsymbol{u}^{(r)} \\
& \boldsymbol{u}_{n}^{(r)}=\boldsymbol{u}_{n}^{(r)}+\Delta \boldsymbol{u}^{(r)}
\end{aligned}
$$

This process is repeated until the residual is smaller than the set convergence criterium and requires us to compute the Jacobian (or iteration) matrix $\boldsymbol{S}^{(r)}$.

$$
\boldsymbol{S}^{(r)}\left(\boldsymbol{u}_{n}^{(r)}\right)=\frac{\partial \boldsymbol{r}^{(r)}}{\partial \boldsymbol{u}^{(r)}}=\frac{\partial \boldsymbol{p}^{(r)}}{\partial \boldsymbol{u}^{(r)}}+\frac{\partial \boldsymbol{p}^{(r)}}{\partial \dot{\boldsymbol{u}}^{(r)}} \frac{\partial \dot{\boldsymbol{u}}^{(r)}}{\partial \boldsymbol{u}^{(r)}}+\boldsymbol{M}^{(r)} \frac{\partial \ddot{\boldsymbol{u}}^{(r)}}{\partial \boldsymbol{u}^{(r)}}+\frac{\partial \boldsymbol{M}^{(r)}}{\partial \boldsymbol{u}^{(r)}} \ddot{\boldsymbol{u}}^{(r)}-\frac{\partial \boldsymbol{f}^{(r)}}{\partial \boldsymbol{u}^{(r)}}+\frac{\partial \boldsymbol{B}^{(r)^{T}} \boldsymbol{\lambda}}{\partial \boldsymbol{u}^{(r)}}
$$

Note that by including the interface forces in the residual (23) and subsequently using this residual to compute the Jacobian matrix (33), the influence of the neighboring substructures is included in the NewtonRaphson iterations. Substituting Eq. (29) into Eq. (33) and only expanding the last term of Eq. (33) shows the contribution of the neighboring substructure in the Jacobian matrix.

$$
\frac{\partial \boldsymbol{B}^{(r)^{T}} \boldsymbol{\lambda}}{\partial \boldsymbol{u}^{(r)}}=\boldsymbol{B}^{(r)^{T}}\left(\boldsymbol{B}^{(s)} \boldsymbol{Y}_{1}^{(s)} \boldsymbol{B}^{(s)^{T}}\right)^{-1} \boldsymbol{B}^{(r)}
$$

This can be physically interpreted as a condensation of the linear substructure into the non-linear substructure. After the nonlinear subsystem is converged, all the (condensed) linear subsystems are updated using the computed interface force.

$$
\boldsymbol{u}_{n}^{(s)}=\tilde{\boldsymbol{u}}_{n}^{(s)}-\frac{d t}{2} \boldsymbol{Y}_{1}^{(s)} \boldsymbol{B}^{(s)^{T}} \boldsymbol{\lambda}_{n}
$$

and a new time step is started. The method for coupling IRFs and nonlinear FE models is shown in figure 2 , where the iteration-loop is enclosed by the doted line.

\section{Computing the coupled response from an initial static equilibrium}

In section III the Impulse Based Substructing (IBS) method has been described as a coupled time integration method. This method needs to be initialized using a set of initial conditions, similar to any other time integration method. In general there are two types of initial conditions one can apply for time simulations. The first being the method of initial accelerations, where one assumes the system is at rest $\left(\dot{\boldsymbol{u}}_{0}=\boldsymbol{O}, \boldsymbol{u}_{0}=\boldsymbol{O}\right)$ and uses the force at the initial time $\left(t_{0}\right)$ to compute the set of initial accelerations.

$$
\boldsymbol{M} \ddot{\boldsymbol{u}}_{0}=\boldsymbol{f}_{0}-\boldsymbol{p}\left(\dot{\boldsymbol{u}}_{0}, \boldsymbol{u}_{0}\right)=\boldsymbol{f}_{0}
$$

As this initial force can be seen as applying an initial impulse to the system, it will result in high initial accelerations and (unwanted) transient effects in the computed responses. The other approach one can apply, is to start from an initial static equilibrium $\left(\boldsymbol{u}_{0} \neq \boldsymbol{O}\right)$.

$$
\boldsymbol{o}=\boldsymbol{f}_{0}-\boldsymbol{p}\left(\dot{\boldsymbol{u}}_{0}=\boldsymbol{O}, \boldsymbol{u}_{0}\right)
$$

As this is a nonlinear problem, one needs to resort to methods for solving nonlinear problems (e.g. NewtonRaphson). Since this method leads to less transient effects from the initialization of the time integration method, it is often preferred for computing the operational behavior of structures and machines.

Hence, this section will present the method for initializing the simulations using an initial static equilibrium. The ones interested in starting the simulations using the initial accelerations are referred to the references. ${ }^{8}$ 


\section{A. Initialization of the IBS method}

In order to start with the time integration scheme as presented in figure 2 , the initial conditions need to be determined as a first step. As was already mentioned, the method will start from an initial static equilibrium. Hence, by substituting $\ddot{\boldsymbol{u}}_{0}=\boldsymbol{O}$ and $\dot{\boldsymbol{u}}_{0}=\boldsymbol{O}$ into Eq. (21), the initial static problem is obtained.

$$
\left\{\begin{array}{l}
\boldsymbol{K}^{(s)} \boldsymbol{u}_{0}^{(s)}=\boldsymbol{f}_{0}^{(s)}-\boldsymbol{B}^{(s)^{T}} \boldsymbol{\lambda}_{0} \\
\boldsymbol{p}^{(r)}\left(\boldsymbol{u}_{0}^{(r)}\right)=\boldsymbol{f}_{0}^{(r)}-\boldsymbol{B}^{(r)^{T}} \boldsymbol{\lambda}_{0} \\
\boldsymbol{B} \boldsymbol{u}_{0}=\boldsymbol{O}
\end{array}\right.
$$

The method for solving this coupled static problem is analog to the approach used for solving the coupled dynamic problem in section III. Firstly, the set of equations for the residual of the nonlinear part is determined.

$$
\boldsymbol{r}^{(s)}\left(\boldsymbol{u}_{0}^{(s)}, \boldsymbol{\lambda}_{0}\right)=\boldsymbol{p}^{(r)}\left(\boldsymbol{u}_{0}^{(r)}\right)+\boldsymbol{B}^{(r)^{T}} \boldsymbol{\lambda}_{0}-\boldsymbol{f}_{0}^{(r)}
$$

The residual is now still a function of the displacements and the interface forces, hence we would like to obtain a relationship expressing the interface forces as a function of the displacements. This can be obtained by substituting the first line of Eq. (38) into the compatibility equation.

$$
\left[\begin{array}{ll}
\boldsymbol{B}^{(s)} & \boldsymbol{B}^{(r)}
\end{array}\right]\left[\begin{array}{l}
\boldsymbol{K}^{(s)^{-1}} \boldsymbol{f}_{0}^{(s)}-\boldsymbol{K}^{(s)^{-1}} \boldsymbol{B}^{(s)^{T}} \boldsymbol{\lambda}_{0} \\
\boldsymbol{u}_{0}^{(r)}
\end{array}\right]=\boldsymbol{O}
$$

Rewriting this equation leads to an expression for the interface forces $\left(\boldsymbol{\lambda}_{0}\right)$.

$$
\boldsymbol{\lambda}_{0}=\left(\boldsymbol{B}^{(s)} \boldsymbol{K}^{(s)^{-1}} \boldsymbol{B}^{(s)^{T}}\right)^{-1}\left[\begin{array}{ll}
\boldsymbol{B}^{(s)} & \boldsymbol{B}^{(r)}
\end{array}\right]\left[\begin{array}{l}
\boldsymbol{K}^{(s)^{-1}} \boldsymbol{f}_{0}^{(s)} \\
\boldsymbol{u}_{0}^{(r)}
\end{array}\right]
$$

By substituting Eq. (41) into Eq. (39), the residual is no longer explicitly dependent on the interface forces $\left(\boldsymbol{\lambda}_{0}\right)$. In order to minimize the residual, the Newton-Raphson approach is used. Therefore we successively solve

$$
\begin{aligned}
& \boldsymbol{S}_{0}^{(r)} \Delta \boldsymbol{u}_{0}^{(r)}=-\boldsymbol{r}^{(r)}\left(\boldsymbol{u}_{0}^{(r)}\right) \\
& \boldsymbol{u}_{0}^{(r)}=\boldsymbol{u}_{0}^{(r)}+\Delta \boldsymbol{u}_{0}^{(r)},
\end{aligned}
$$

where $\boldsymbol{S}_{0}^{(r)}$ is the iteration (or Jacobian) matrix, until the residual is smaller than a chosen threshold or convergence criterion. The iteration matrix $\boldsymbol{S}_{0}^{(r)}$ is obtained for the static case by computing the derivative of the residual with respect to the displacements.

$$
\boldsymbol{S}_{0}^{(r)}=\frac{\partial \boldsymbol{r}^{(r)}}{\partial \boldsymbol{u}^{(r)}}=\frac{\partial \boldsymbol{p}^{(r)}}{\partial \boldsymbol{u}^{(r)}}-\frac{\partial \boldsymbol{f}^{(r)}}{\partial \boldsymbol{u}^{(r)}}+\frac{\partial \boldsymbol{B}^{(r)^{T}} \boldsymbol{\lambda}}{\partial \boldsymbol{u}^{(r)}}
$$

Assuming here that the external load is independent of the displacements of the structure, this results in the following iteration matrix.

$$
\boldsymbol{S}_{0}^{(r)}=\frac{\partial \boldsymbol{p}_{0}^{(r)}}{\partial \boldsymbol{u}_{0}^{(r)}}+\boldsymbol{B}^{(r)^{T}}\left(\boldsymbol{B}^{(s)} \boldsymbol{K}^{(s)^{-1}} \boldsymbol{B}^{(s)^{T}}\right)^{-1} \boldsymbol{B}^{(r)}
$$

Analog to the iteration matrix used in section III, it is clear that the stiffness of the neighboring linear substructures is condensed in the iteration matrix of the nonlinear part. Hence, in Eq. (42) we iterate on the global problem and thus finds the global $\boldsymbol{u}_{0}^{(r)}$. Substituting the obtained displacements into Eq. (41) gives the interface forces, which after substitution into the first line of Eq. (38), leads to the set of displacements for all the linear substructures that are described in their IRFs. The now obtained displacements serve as the initial conditions for the time integration scheme given in figure 2 . 


\section{B. Obtaining the static flexibility matrix}

In the previous subsection the stiffness matrix of the linear subsystem is used to find the initial static equilibrium. But, as there is a relation between the stiffness matrix and the IRFs, the stiffness matrix is not required in order to find this static equilibrium. If we would apply a constant (unit) force in the convolution product given in Eq. (4), we would obtain

$$
\boldsymbol{u}(t)=\int_{\tau=0}^{t} \boldsymbol{Y}(t-\tau) \mathbf{1}_{j} d \tau,
$$

where $\mathbf{1}_{j}$ is a vector with a unit coefficient for dof $j$. If $t$ goes to infinity, the dynamic response will damp out and only the static response remains.

$$
\boldsymbol{u}(\infty)=\int_{\tau=0}^{\infty} \boldsymbol{Y}(t-\tau) \mathbf{1}_{j} d \tau=\boldsymbol{K}^{-1} \mathbf{1}_{j}
$$

Hence, we can obtain the static flexibility matrix $\boldsymbol{K}^{-1}$ by simply integrating the IRFs from $t=0$ to infinity.

$$
\boldsymbol{K}^{-1}=\int_{\tau=0}^{\infty} \boldsymbol{Y}(\tau) d \tau
$$

By discretizing the continuous convolution product given in Eq. (47) and assuming that we can truncate it after $k+1$ timesteps, we can directly find the static flexibility matrix.

$$
\boldsymbol{K}^{-1} \approx d t \sum_{i=0}^{k} \boldsymbol{Y}_{i}
$$

Substituting of Eq. (48) into Eq. (38) results in

$$
\left\{\begin{array}{l}
\boldsymbol{u}_{0}^{(s)}=d t \sum_{i=0}^{k} \boldsymbol{Y}_{i}\left(\boldsymbol{f}_{0}^{(s)}-\boldsymbol{B}^{(s)^{T}} \boldsymbol{\lambda}_{0}\right) \\
\boldsymbol{p}^{(r)}\left(\boldsymbol{u}_{0}^{(r)}\right)=\boldsymbol{f}_{0}^{(r)}-\boldsymbol{B}^{(r)^{T}} \boldsymbol{\lambda}_{0} \\
\boldsymbol{B} \boldsymbol{u}_{0}=\boldsymbol{o}
\end{array}\right.
$$

and can be used to determine the initial static equilibrium, as was described in subsection A. Note that this is relatively cheap, as the summation given in Eq. (48) needs to be done only once and no factorization is required in Eq. (49).

\section{Application to an Offshore Wind Turbine}

In order to show the effectiveness of the method, a case study has been selected. In this case study, the structural model of the NREL 5MW turbine ${ }^{9}$ has been taken and coupled to the UpWind reference jacket model, ${ }^{10}$ which was also used in the OC4 benchmark study. ${ }^{11}$ Both models are shown in figure 3 . The wind turbine is modeled as a nonlinear finite element model, where the nonlinearity arises from the nonlinear damper that is attached to the hub-node. This damper is added for simulating an aerodynamic coupling, even though this damper is far from accurately describing the actual aerodynamic behavior, it demonstrates that the method can handle nonlinearities. The wind loading is represented by randomly distributing a random force and a constant force over the entire rotor. The UpWind jacket is modeled using impulse response functions that were computed for the interface DoF, together with the IRFs due to the distributed wave loading, as is described in section II. In this section, two different load cases are shown. The first load case is one where only the equivalent wind loading is applied over the rotor and it is shown in subsection A. For the second load case (subsection B), equivalent wind loading is applied over the rotor and the offshore jacket is excited by wave loads. In both cases the results obtained from the IBS method are compared to those of a Newmark time integration performed on the full, non-substructured, model of the offshore wind turbine, comprising of the jacket and wind turbine. 


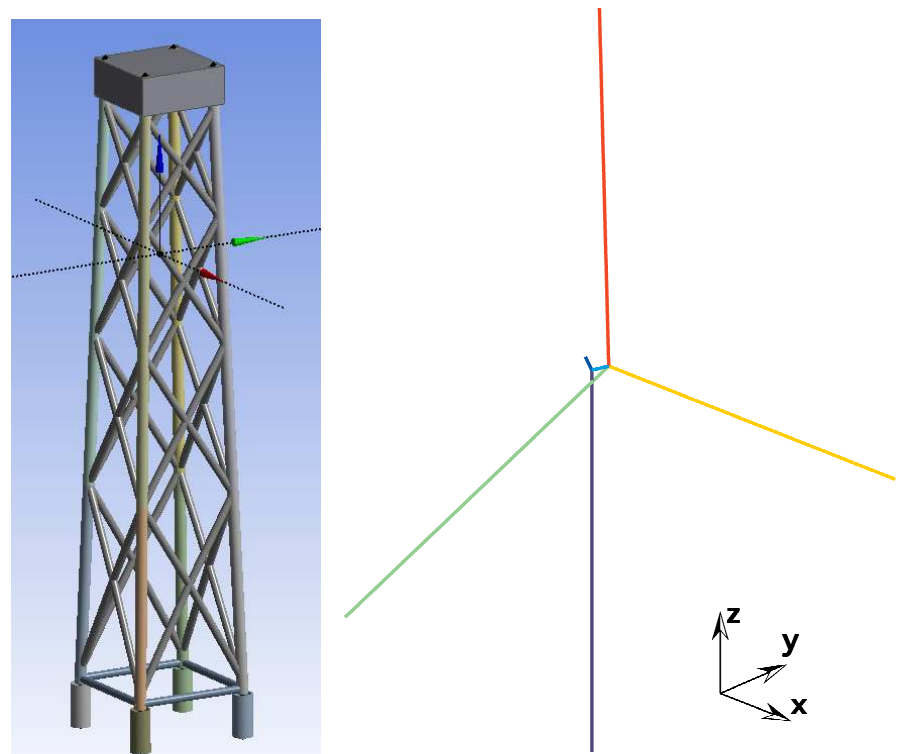

Figure 3. Models of the Upwind reference jacket (left) and the NREL 5MW turbine (right)

\section{A. Wind loading on the rotor}

Firstly, we look at the case where only the equivalent wind loading is applied. As the wind loading in the case of the substructuring analysis is exactly equal to the loading applied on the full model, the accuracy of the IBS method can be evaluated from the obtained results. In figure 4 it can be seen that the method

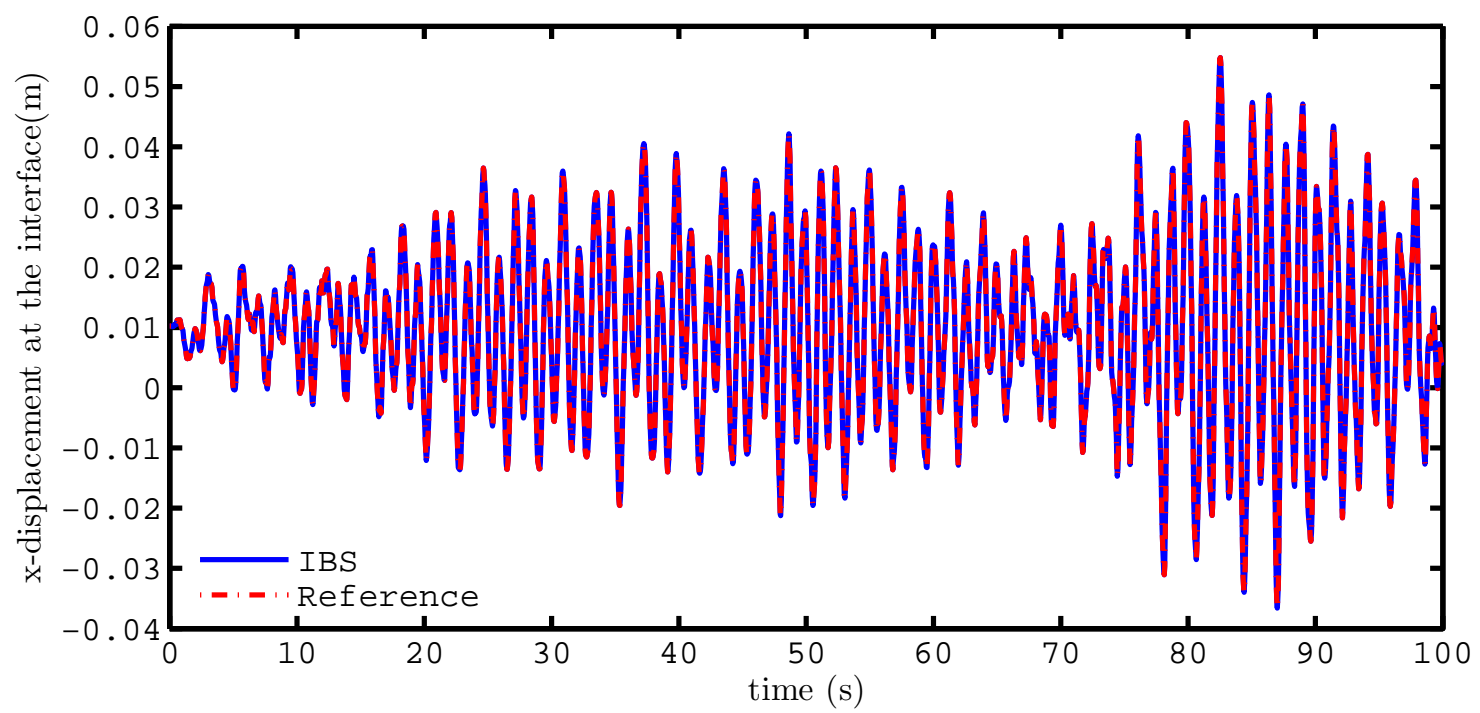

Figure 4. Comparison of time responses obtained by IBS and full Newmark time integration, wind loading only

retrieves the same results as for the non-substructured model. The time response of the reference model, which is the full FE model of the jacket and turbine combined, shows a perfect match with the time response computed using the Impulse Based Substructuring method. The relative error is shown in figure 5, which is computed according to Eq. (50).

$$
\epsilon_{u}(t)=\frac{\left\|u_{r e f}(t)-u_{I B S}(t)\right\|}{\left\|u_{r e f}(t)\right\|}
$$




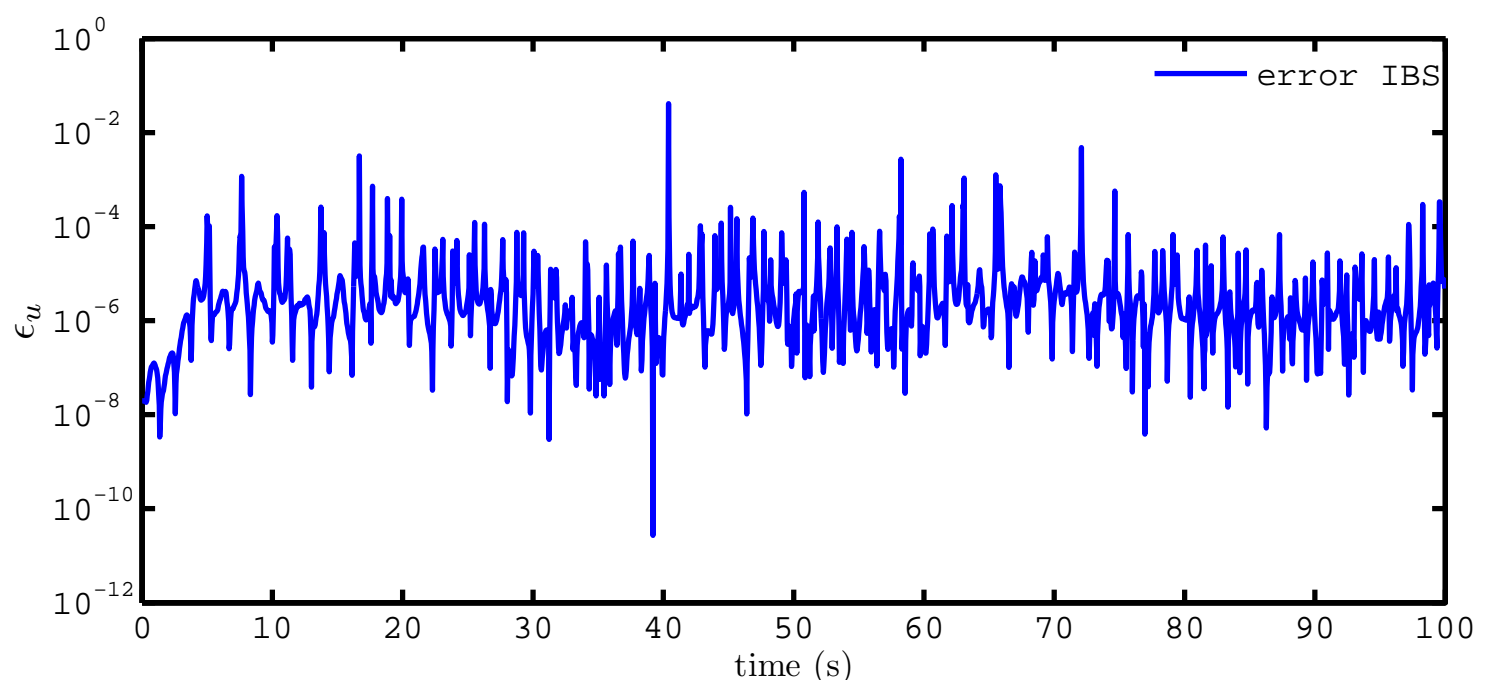

Figure 5. Relative error $\epsilon_{u}$ in the x-direction, wind loading only

From figure 4 it can be seen that, although very small, the error is not equal to zero. This can be explained from the fact that in the Newton-Raphson iterations the residual (error on the equilibrium) is minimized up to a certain tolerance (in this case $\left\|\boldsymbol{r}_{n}\right\|<10^{-6}\left\|\boldsymbol{p}_{n}-\boldsymbol{B}^{T} \boldsymbol{\lambda}\right\|$ ). Hence, by setting this tolerance more strict, the errors on the displacements become smaller. As the dynamic behavior represented by the Impulse Response Functions is numerically exact, the exact same result would be obtained if we would iterate up to the point where the residual is zero. Note that in practice this can never be achieved due to numerical errors.

\section{B. Combined wind and wave loading}

For the second load case, the jacket structure is excited by sea waves, which are assumed to be irregular (linear) waves with a significant wave height of $9.40 \mathrm{~m}$, and a current with speeds up to $1.2 \mathrm{~m} / \mathrm{s}^{2}$ at the mean sea level. ${ }^{10}$ The wave forces are computed beforehand, which is done by assuming small velocities of the structure, hence discarding the relative velocity terms in the Morrison equation. ${ }^{12}$ The wave forces have

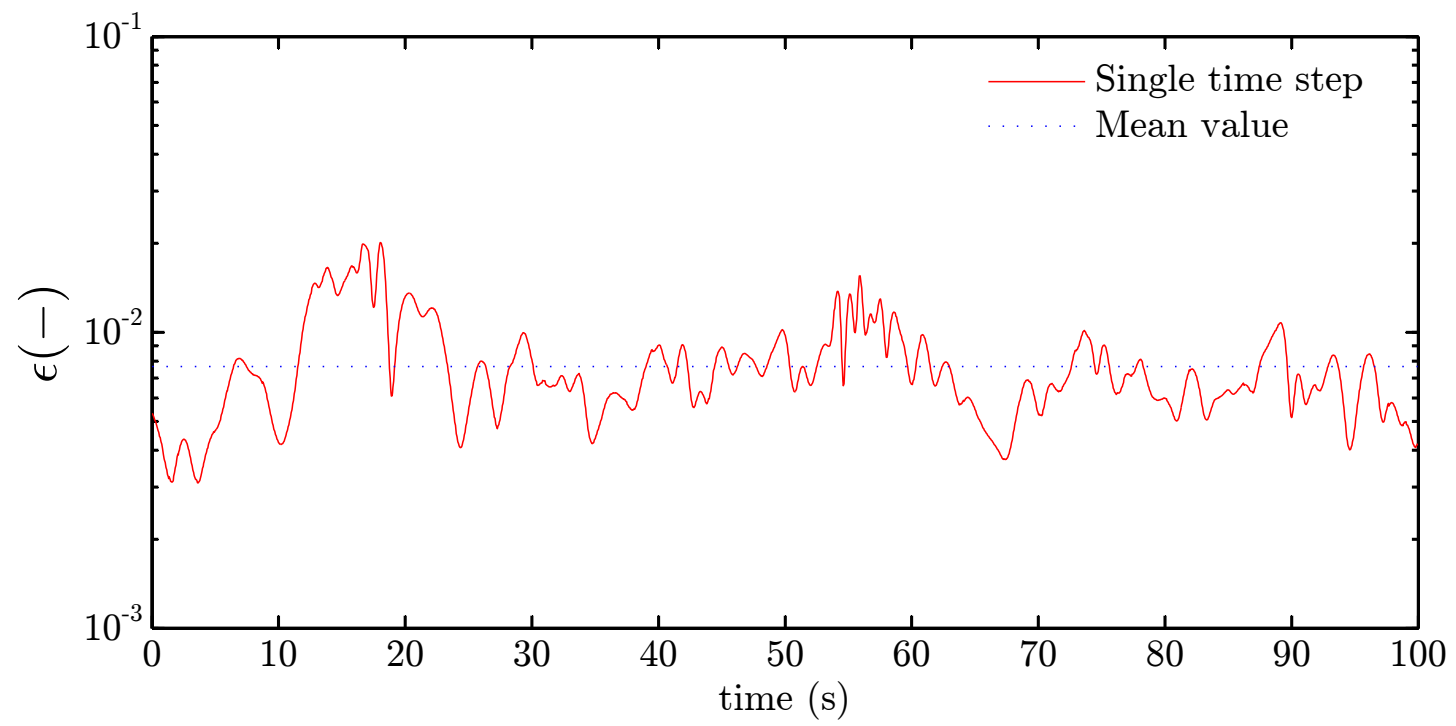

Figure 6. Norm of the force error when using a POD representation with 15 modes. 
been reduced using fifteen force shape distributions over the structure, as was presented in section II. This reduces the vector of applied forces from $p$-entries, related to the DoF of the physical model $(p=1062)$, to only a limited number of distributed forces $\left(p_{\phi}=15\right)$. The space distributions have been determined using a proper orthogonal decomposition (POD) analysis on the force time series, where only the 15 most important proper orthogonal modes (POMs) where retained. Using the force space distributions (which we will call "modes" from now on and are assumed to be normalized), the modal force amplitudes $(\boldsymbol{\eta})$, as presented in Eq. (12), are computed according to:

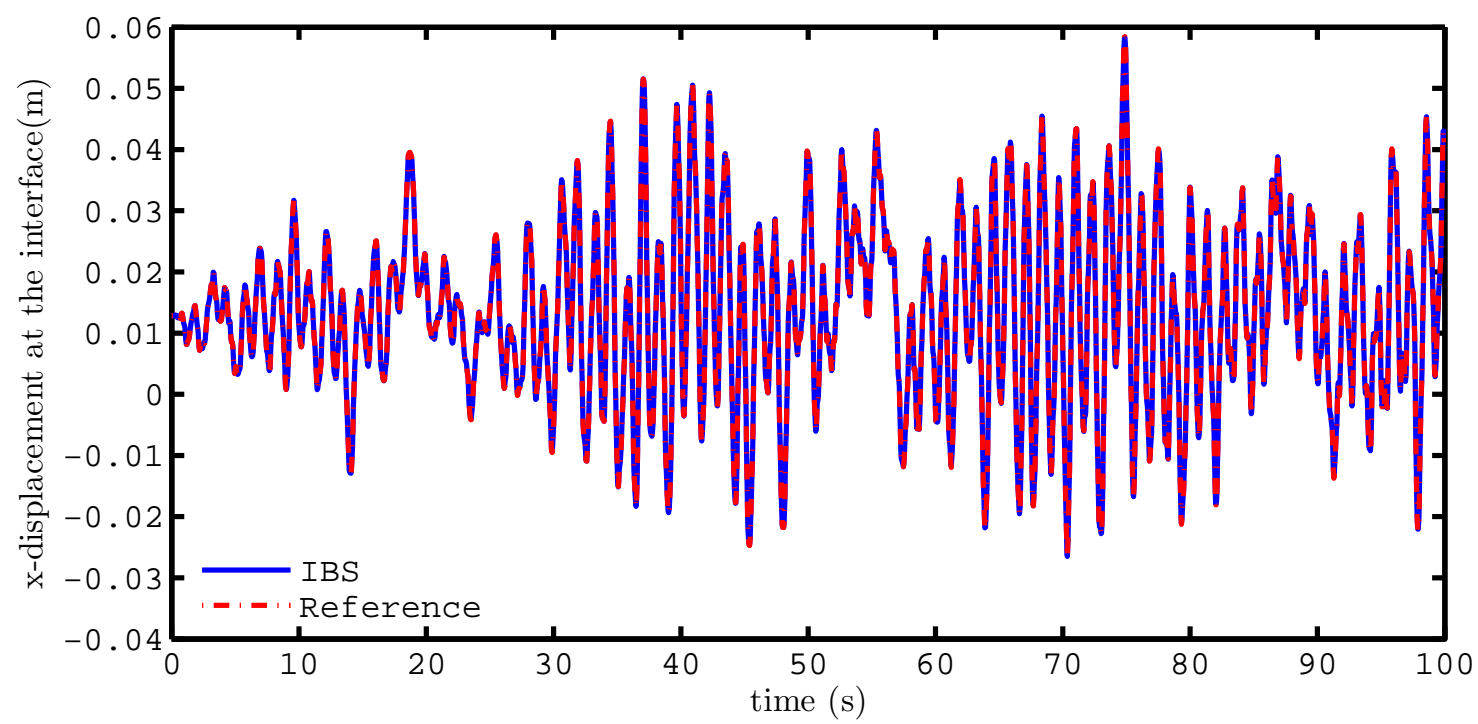

Figure 7. Comparison of time responses obtained by IBS and full Newmark time integration, wind and wave loading

$$
\boldsymbol{\eta}(t)=\boldsymbol{\Phi}^{T} \boldsymbol{f}(t),
$$

which can be expanded back to physical forces by projecting them on the set of retained modes

$$
\tilde{\boldsymbol{f}}(t)=\boldsymbol{\Phi} \boldsymbol{\Phi}^{T} \boldsymbol{f}(t) .
$$

By taking the L2-norm (per time step) of the differences between the original applied forces $(\boldsymbol{f}(t))$ and the

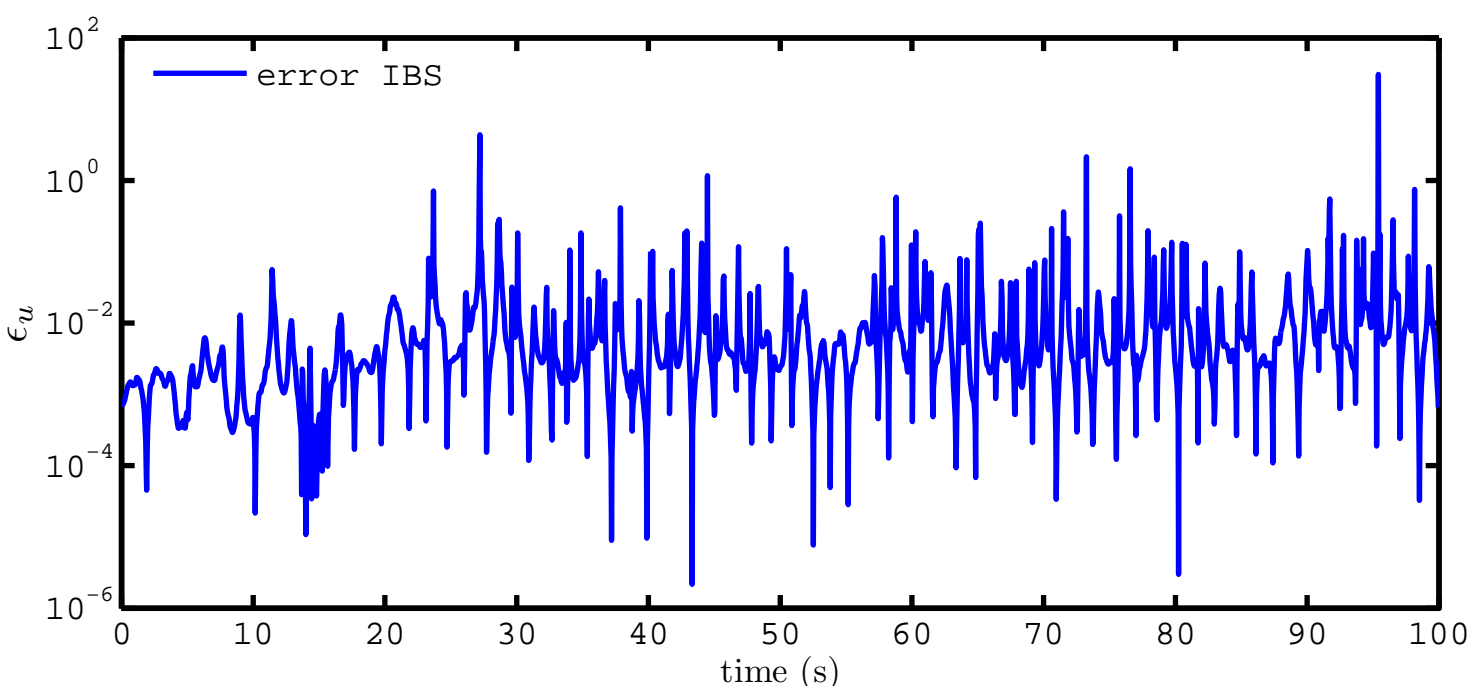

Figure 8. Relative error $\epsilon_{u}$ in the x-direction, wind and wave loading 
reduced forces $(\tilde{\boldsymbol{f}}(t))$ and dividing this by the L2-norm of the original applied forces, a qualitative measure $(\epsilon(t))$ is found for the amount of force "lost" due to the reduction step,

$$
\epsilon(t)=\frac{\left\|\boldsymbol{f}(t)-\boldsymbol{\Phi} \boldsymbol{\Phi}^{T} \boldsymbol{f}(t)\right\|}{\|\boldsymbol{f}(t)\|}
$$

The result is shown in figure 6. It can be seen that after the reduction step (on average) $99.2 \%$ of the L2-norm of the force was retained. But it should be noted that locally a larger error can exist, which could cause errors on the computed response of the structure. The results from the time integrations are shown in figure 7, which again appear to be a nearly perfect match. If figures 5 and 8 are compared to each other, we note that the error, computed using Eq. (50), made on the displacements is slightly bigger. This comes from the fact that the reduced wave loads are an approximation of the original time series of wave loading. Nevertheless, this clearly demonstrates that the idea of using an impulse response due to a distributed load, can be easily applied within the IBS framework and can produce excellent results.

\section{Conclusions}

From this work it was shown that the Impulse Based Substructuring method can be applied to couple any type of (linearized) offshore structure to a wind turbine in order to compute the coupled dynamic response. The method can be easily implemented, as it only requires a number of small augmentations to the standard Newmark time integration scheme for non-linear systems, combined with a simple scheme to solve the discretized Duhamel integral. From the figures it can be seen that the achievable accuracy is in the same order as the convergence criteria set for the Newton-Raphson iterations.

In addition it is shown that the method is able to compute the time response of structures which start from non-zero initial conditions. Therefore, the time integration can be initialized from a static equilibrium, hence eliminating any unwanted transient effects at the start of the time integration.

Finally, it is shown that the Impulse Based Substructuring method can also be applied to structures which have relatively large force loaded areas. This is done by allowing one to compute the impulse responses due to a distributed load. Combining this idea with proper orthogonal decomposition methods in order to find the predominant force distribution shapes gives an efficient and accurate method for including wave loading on an offshore structure in the analysis.

\section{References}

${ }^{1}$ Guyan, R., "Reduction of Stiffness and Mass Matrices," AIAA Journal, Vol. 3, February 1965, pp. 380.

${ }^{2}$ Seidel, M., von Mutius, M., Rix, P., and Steudel, D., "Integrated analysis of wind and wave loading for complex support structures of Offshore Wind Turbines," Conference Proceedings Offshore Wind 2005, Copenhagen, 2005.

${ }^{3}$ Passon, P., "Design of Offshore Wind Turbine Foundations in Deeper Water," TORQUE 2010: The Science of Making To1rque from Wind, June 28-30, Crete, Greece, 2010.

${ }^{4}$ Rixen, D., "Substructuring using Impulse Response Functions for Impact Analysis," Proceedings of the IMAC - XXVIII, Jacksonville, Florida USA, 2010.

${ }^{5}$ Géradin, M. and Rixen, D., Mechanical Vibrations - Theory and Application to Structural Dynamics, John Wiley \& Sons, Chichester, England, December 1997.

${ }^{6}$ de Klerk, D., Rixen, D., and Voormeeren, S., "General Framework for Dynamic Substructuring: History, Review and Classification of Techniques," AIAA Journal, Vol. 46, No. 5, May 2008, pp. 1169-1181.

${ }^{7}$ Newmark, N., "A Method of Computation For Structural Dynamics," Proceedings of the American Society of Civil Engineers, Journal of the Engineering Mechanics Division, 1959, pp. 67-94.

${ }^{8}$ van der Valk, P. and Rixen, D., "An Effective Method for Assembling Impulse Response Functions to Linear and NonLinear Finite Element Models," Proceedings of the SEM IMAC XXX Conference Jan. 30 - Feb. 2, 2012, Jacksonville, FL USA, 2012.

${ }^{9}$ Jonkman, J., Butterfield, S., Musial, W., and Scott, G., "Definition of a 5-MW Reference Wind Turbine for Offshore System Development," Tech. rep., National Renewables Energy Laboratory, 2009.

${ }^{10}$ de Vries, W., Vemula, N. K., Passon, P., Fischer, T., Kaufer, D., Matha, D., Schmidt, B., and Vorpahl, F., "Final report WP 4.2 - Support Structure Concepts for Deep Water Sites," Tech. rep., Project UpWind, 2011.

${ }^{11}$ Vorpahl, F., Popko, W., and Kaufer, D., "Description of a basic model of the "UpWind reference jacket" for code comparison in the OC4 project under IEA Wind Annex XXX," Tech. rep., Fraunhofer Institute for Wind Energy and Energy System Technology (IWES), 2011.

${ }^{12}$ Morison, J. R., O'Brien, M. P., Johnson, J. W., and Schaaf, S. A., "The force exerted by surface waves on piles," Petroleum Transactions (American Institute of Mining Engineers), Vol. 189, 1950, pp. 149-154. 